\title{
Traitement à l'ivermectine pour l'infection à Strongyloides chez les patients atteints de COVID-19
}

\author{
Elizabeth Leung ${ }^{1,2,3 *}$, Sumit Raybardhan ${ }^{3,4}$, Christopher Graham ${ }^{3,5}$, Caroline Nott ${ }^{3,6}$, \\ Bradley J Langford ${ }^{3,7}$, Nisha Andany ${ }^{3,8,9}$, William Ciccotelli, ${ }^{3,10,11}$, Sumon Chakrabarti, \\ Isaac I Bogoch 9 , Wayne L Gold ${ }^{3,9,12}$, Menaka Pai ${ }^{3,13,14,15}$, Andrew M Morris $3,9,15,16$, Anne McCarthy ${ }^{17}$
}

\section{Résumé}

L'ivermectine, un agent antiparasitaire, n'est pas recommandé pour la prophylaxie ou le traitement de la maladie à coronavirus 2019 (COVID-19). L'utilisation inappropriée de l'ivermectine pour le traitement de la COVID-19 peut en diminuer la disponibilité pour les patients atteints d'infections parasitaires graves qui pourraient en bénéficier et aggraver la pénurie actuelle de ce médicament au Canada. Cependant, les patients atteints de COVID-19 qui sont des candidats pour recevoir des thérapies immunomodulatrices (e.g. corticostéroïdes et inhibiteurs de l'interleukine-6) peuvent être exposés au risque de syndrome d'hyperinfection et de la maladie disséminée à Strongyloides stercoralis. Ces complications peuvent être graves, voire mortelles. Il est important de reconnaître et de dépister les patients qui présentent des risques de strongyloïdose, car ces patients peuvent avoir besoin d'un traitement à l'ivermectine pour éviter le risque d'un syndrome d'hyperinfection et d'une maladie disséminée, qui est souvent mortelle. Les médecins devraient suivre des recommandations fondées sur des données probantes pour dépister et traiter l'infection à Strongyloides chez les patients atteints de COVID-19 qui pourraient recevoir des traitements propres à la COVID-19 qui modifient la réponse immunitaire et qui peuvent entraîner un syndrome d'hyperinfection ou une maladie disséminée.

Citation proposée : Leung E, Raybardhan S, Graham C, Nott C, Langford BJ, Andany N, Ciccotelli W, Chakrabarti S, Bogoch II, Gold WL, Pai M, Morris AM, McCarthy A. Traitement à l'ivermectine pour l'infection à Strongyloides chez les patients atteints de COVID-19. Relevé des maladies transmissibles au Canada 2021;47(7/8):348-54. https://doi.org/10.14745/ccdr.v47i78a04f

Mots-clés : COVID-19, Strongyloides, ivermectine, immunosuppression, corticostéroïdes, inhibiteurs de l'IL-6, strongyloïdose

\section{Introduction}

L'ivermectine est un médicament oral approuvé au Canada pour le traitement de certaines infections parasitaires (e.g. la strongylö̈dose et l'onchocercose) (1). Des données in vitro ont démontré que l'ivermectine a une activité antivirale contre le coronavirus du syndrome respiratoire aigu sévère 2 (SRAS-CoV-2) et peut empêcher les protéines virales de se lier aux cellules et d'y pénétrer (2). Cela a contribué à l'opinion selon laquelle l'ivermectine peut avoir des effets antiviraux in vivo. Cependant, les concentrations plasmatiques requises pour obtenir l'effet antiviral sont considérablement plus élevées que les concentrations plasmatiques maximales atteignables avec des doses tolérables chez l'humain (3). L'ivermectine n'est actuellement pas recommandée pour la prophylaxie ou le traitement de la maladie à coronavirus 2019 (COVID-19) (4), car les données actuelles ne montrent aucun avantage clinique (5). En l'absence de données probantes démontrant un avantage clinique de l'ivermectine dans le traitement de la COVID-19, il existe des raisons impérieuses de conserver des approvisionnements limités d'ivermectine à l'échelle nationale et provinciale en ne l'utilisant que pour des indications fondées sur des données probantes.
Cette oeuvre est mise à la disposition selon les termes de la licence internationale Creative Commons Attribution 4.0

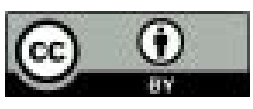

Affiliations

1 Department of Pharmacy, St. Michael's Hospital/Unity Health Toronto, Toronto, ON

${ }^{2}$ Leslie Dan Faculty of Pharmacy, University of Toronto, Toronto, ON

${ }^{3}$ Ontario COVID-19 Science Advisory Table Drugs and Biologics Clinical

Practice Guidelines Working Group

${ }^{4}$ Department of Pharmacy, North York General Hospital, Toronto, ON

${ }^{5}$ Trillium Health Partners,

Mississauga, ON

${ }^{6}$ Department of Medicine, University of Ottawa, Ottawa, ON

7 Public Health Ontario, Toronto, ON

${ }^{8}$ Division of Infectious Diseases,

Sunnybrook Health Sciences Centre,

Toronto, ON

${ }^{9}$ Department of Medicine, University of Toronto, Toronto, ON

${ }^{10}$ Grand River Hospital, Kitchener, ON

${ }^{11}$ St. Mary's General Hospital, Kitchener, ON

12 Department of Medicine, University Health Network, Toronto, ON

${ }^{13}$ McMaster University, Hamilton, ON

${ }^{14}$ Hamilton Health Sciences and Hamilton Regional Laboratory Medicine Program, Hamilton, ON

${ }^{15}$ Ontario COVID-19 Science Advisory Table

${ }^{16}$ Department of Medicine, Sinai Health and University Health Network, Toronto, ON

${ }^{17}$ Division of Infectious Diseases, The Ottawa Hospital, Ottawa, ON

*Correspondance :

beth.leung@utoronto.ca 
Il est important de noter que l'utilisation primaire et approuvée de l'ivermectine est en tant qu'agent antiparasitaire. Un parasite d'intérêt est Strongyloides stercoralis. L'infection à Strongyloides varie au niveau de la présentation, allant de l'infection intestinale asymptomatique à l'hyperinfection et à la maladie disséminée grave. Les patients atteints d'une infection asymptomatique chronique à $S$. stercoralis et d'une insuffisance immunitaire peuvent développer une "auto-infection accélérée», connue sous le nom d'hyperinfection. Ces patients peuvent également avoir une maladie disséminée secondaire à la migration larvaire à l'extérieur du réservoir anatomique habituel, accompagnée de présentations variées (e.g. pneumonie, infection du système nerveux central, bactériémie et septicémie récurrentes à Gram négatifs ou polymicrobiennes). L'immunosuppression, en particulier l'utilisation de corticostéroïdes, a été associée à un risque accru de syndrome d'hyperinfection chez les patients atteints d'infection chronique à Strongyloides.

Les médecins peuvent consulter l'exposé scientifique de la Table de consultation scientifique de l'Ontario intitulé «Ivermectin as Empiric Treatment for Strongyloides in Patients with COVID-19 Disease " pour obtenir de plus amples renseignements sur les recommandations présentées dans cet article. L'exposé complet de la Table scientifique est accessible en ligne (en anglais seulement).

\section{Situation actuelle}

Chez les patients atteints de strongylö̈dose (y compris l'infection asymptomatique à Strongyloides), l'immunosuppression peut entraîner une aggravation de l'infection parasitaire, y compris l'hyperinfection et la maladie disséminée, qui est une maladie dévastatrice ayant des taux de mortalité approchant $90 \%$ si elle n'est pas traitée $(6,7)$. Même si la plupart des signalements de syndrome d'hyperinfection impliquent des corticostéroïdes comme facteur de risque principal, il y a aussi des signalements avec d'autres agents immunosuppresseurs ou immunomodulateurs, y compris le facteur de nécrose tumorale, I'interleukine-1 (IL-1) et d'autres agents non stéroïdiens qui réduisent le nombre des lymphocytes $(6,7)$. Un récent examen de l'European Society of Clinical Microbiology and Infectious Diseases sur l'innocuité des thérapies biologiques n'a pas indiqué que Strongyloides était un risque infectieux important avec les inhibiteurs de l'interleukine-6 (IL-6) (y compris le tocilizumab) (8). Cependant, il y a eu des cas d'hyperinfection à Strongyloides qui se développent chez des patients qui ont reçu un traitement aux corticostéroïdes concomitant au moyen du tocilizumab (9-11).

La dexaméthasone et le tocilizumab, deux thérapies fondées sur des données probantes pour le traitement de patients atteints de COVID-19 modérée ou grave, sont tous deux des anti-inflammatoires ayant des effets immunosuppresseurs. II n'y a pas de relation claire entre une dose seuil et la durée de l'immunosuppression à laquelle le risque de strongylö̈dose disséminée peut se produire. En ce moment, la façon dont les médicaments immunosuppresseurs concomitants peuvent interagir pour avoir une incidence sur le risque d'hyperinfection ou sur la possibilité que les personnes qui reçoivent de multiples thérapies immunosuppressives présentent un risque supplémentaire de maladie disséminée n'en sont pas bien décrites.

\section{Approvisionnement limité actuel d'ivermectine}

L'ivermectine est un médicament essentiel pour le traitement de la strongyloïdose (et c'est une composante importante de la thérapie combinée pour le syndrome d'hyperinfection et la maladie fulminante et disséminée). En date du 21 janvier 2021, l'ivermectine a été inscrite sur la liste des pénuries de médicaments par Pénuries de médicaments Canada, avec seulement des quantités limitées disponibles en raison de l'augmentation de la demande pour le médicament; la date de fin prévue de la pénurie est le 31 décembre 2021, laquelle est sujette à changement en fonction de l'offre et de la demande (12). Par conséquent, les médecins sont actuellement encouragés à utiliser l'ivermectine seulement lorsque cela est nécessaire pour des indications cliniques fondées sur des données probantes et ils pourraient devoir être prêts à envisager d'utiliser des agents de substitution si l'ivermectine est indisponible localement. Dans ce bref examen, nous discutons de la mise en œuvre des recommandations existantes de dépistage du Strongyloides chez les patients atteints de COVID-19 et nous suggérons des stratégies de traitement de ce parasite pour préserver l'approvisionnement en ivermectine tout en assurant un traitement approprié pour les patients atteints de COVID-19 à risque d'hyperinfection (voir l'appendice tableau A1).

\section{Évaluation du risque de Strongyloides chez les patients atteints de COVID-19}

Le taux d'infection à Strongyloides au Canada n'est pas bien décrit, mais le Comité national de conseils en matière de médecine tropicale et de voyages (CCMTMV) estime qu'au Canada, au moins 2,5 millions de personnes sont atteintes d'une strongyloïdose intestinale (en supposant un taux de prévalence de $40 \%$ dans le pays d'origine du patient, même si la séroprévalence est supérieure à $60 \%$ chez les immigrants des régions endémiques). En 2016, le CCMTMV a noté que près de sept millions de Canadiens étaient nés à l'étranger et que $85 \%$ de cette population venait d'un pays où $S$. stercoralis est endémique. Par conséquent, le risque de strongyloïdose au Canada n'est pas négligeable (6).

Pour les patients atteints de COVID-19 qui pourraient recevoir un traitement immunosuppresseur, la première étape consiste à évaluer leur risque de développer une strongyloïdose en fonction de facteurs épidémiologiques et cliniques. Nous recommandons de suivre l'étape 1 des lignes directrices du CCMTMV_évaluation épidémiologique (selon le pays de résidence antérieure ou l'exposition prolongée) $(6,13)$. II convient de noter que les lignes directrices du CCMTMV décrivent le 
risque lié à une dose de corticostéroïde «équivalant à $20 \mathrm{mg} / \mathrm{jour}$ de prednisone pendant au moins deux semaines » et nous supposons que la dose de corticostéroïde recommandée pour le traitement de la COVID-19 est similaire à cette limite relativement arbitraire. Le syndrome d'hyperinfection a été signalé avec une gamme de doses et de durées de corticostéroïdes, et nous ignorons encore si les traitements immunosuppresseurs concomitants contribuent à un risque relatif plus élevé d'hyperinfection.

Nous reconnaissons également que le risque épidémiologique géographique seul peut placer de nombreux patients dans la catégorie de risque modéré (sinon la catégorie de risque élevé) et que ces données peuvent également chevaucher les données démographiques des patients dans les quartiers et les collectivités qui ont été touchés fortement et de façon disproportionnée par la pandémie de COVID-19 (14).

Nous recommandons également de suivre l'étape 2 des lignes directrices du CCMTMV— " évaluation du risque clinique et du syndrome clinique suspect ». Cette étape permettra de déterminer les analyses diagnostiques à effectuer pour détecter la présence de strongyloïdose. Idéalement, le test de dépistage de $S$. stercoralis est effectué avant l'administration du traitement immunosuppresseur, mais souvent cela est impossible chez les patients atteints de COVID-19. Les patients présentant un risque épidémiologique modéré ou élevé $d$ 'infection à Strongyloides devraient procéder à des analyses sérologiques dès que possible (même si l'immunosuppression a déjà été amorcée), tandis que les patients présentant un faible risque épidémiologique d'infection à Strongyloides n'ont pas besoin d'un dépistage diagnostique à moins qu'il y ait des soupçons cliniques d'hyperinfection ou de strongylö̈dose disséminée. Les médecins doivent noter que tout patient qui présente des signes ou des symptômes cliniques de strongyloïdose active, d'hyperinfection ou de diffusion nécessitera des tests diagnostiques supplémentaires, un traitement empirique et une consultation auprès d'experts (6).

\section{Traitement de la strongyloïdose chez les patients atteints de la COVID-19}

La dose d'ivermectine pour le traitement de la strongyloïdose varie en fonction du syndrome clinique. Les formes moins graves de strongyloïdose (e.g. la strongyloïdose intestinale légère ou asymptomatique) sont maintenant traitées principalement avec une dose unique d'ivermectine en fonction du poids (200 $\mu \mathrm{g}$ par kilogramme), fondée sur des données probantes émergentes selon lesquelles une seule dose est aussi efficace que de multiples doses (15). Les médecins peuvent envisager de répéter la sérologie après un schéma posologique unique s'ils soupçonnent que le traitement est un échec. Lorsqu'un schéma posologique à deux doses d'ivermectine est utilisé, les doses sont généralement administrées pendant des jours consécutifs ou séparées par un intervalle de 14 jours. Lorsqu'un schéma posologique à deux doses est administré, le schéma posologique à dose séparée est préférable aux doses consécutives en raison du risque d'infection prépatent découlant de l'auto-infection, même si des doses consécutives peuvent être utilisées si l'adhésion aux médicaments est préoccupante (e.g. les difficultés du système de santé liées à la fréquence accrue des transferts intrahospitaliers et interhospitaliers pendant la pandémie de COVID-19). Les formes graves de strongyloïdose (e.g. hyperinfection et infection à Strongyloides disséminée) nécessitent souvent une dose quotidienne d'ivermectine en fonction du poids, parfois administrée en association avec $d^{\prime}$ autres agents antihelminthiques comme l'albendazole (6).

Nous recommandons de suivre les lignes directrices de l'étape 3 du CCMTMV pour décider si un patient doit être examiné et traité au moyen de l'ivermectine pour la strongyloïdose. Cette approche tient compte à la fois des évaluations épidémiologiques (étape 1) et des risques cliniques (étape 2). La plupart des patients présentant un risque épidémiologique d'infection à Strongyloides et pour lesquels des thérapies immunomodulatrices (soit dexaméthasone, soit tocilizumab, ou les deux) sont prises en considération pendant leur hospitalisation pour la COVID-19, sont à risque de contracter une strongylö̈dose asymptomatique. Comme mentionné précédemment, bon nombre de ces patients peuvent également se retrouver dans la catégorie du risque épidémiologique géographique «élevé» ou «modéré». Dans ces cas, il est important que les médecins envoient les échantillons appropriés pour les tests diagnostiques et qu'ils surveillent également le patient après qu'il ait reçu un traitement immunomodulateur pour détecter tout signe ou symptôme d'infection à Strongyloides qui pourrait potentiellement progresser vers le syndrome d'hyperinfection et propager la maladie si elle n'est pas traitée rapidement.

Les lignes directrices du CCMTMV (avant la COVID-19) indiquent que le traitement empirique de deux doses d'ivermectine peut être administré «dans les rares cas où le patient est considéré comme présentant un risque élevé de strongyloïdose et d'immunosuppression, et ne peut attendre le test diagnostique définitif» (6). Toutefois, étant donné l'offre nationale limitée actuelle d'ivermectine, nous recommandons d'attendre les résultats de la sérologie pour Strongyloides (si les résultats peuvent être obtenus assez rapidement, e.g. dans les 24 heures) avant de débuter l'ivermectine pour le traitement de la strongyloïdose asymptomatique. Les patients ayant un résultat sérologique pour Strongyloides réactif ou indéterminé devraient être traités pour la strongyloïdose asymptomatique, comme décrit précédemment. Si les résultats de laboratoire ne peuvent être accélérés, il est raisonnable d'attendre les résultats de la sérologie si le patient est cliniquement stable et de n'administrer qu'un traitement empirique à l'ivermectine si le patient est cliniquement instable. S'il y a des soupçons d'hyperinfection au moment de la présentation de la COVID-19, il est recommandé de consulter un spécialiste des maladies infectieuses. 
Enfin, un point important à considérer avant le début d'un traitement à l'ivermectine est le dépistage de la présence concomitante de loase (infection par le nématode filaire Loa loa), en raison du risque de réactions graves, y compris l'encéphalopathie fatale, si l'ivermectine est administrée à un patient atteint de loase non traitée. Les médecins devraient consulter un expert en maladies infectieuses et en médecine tropicale pour obtenir des recommandations sur le traitement de Strongyloides chez les patients provenant de régions endémiques de Loa loa, c'est-à-dire l'Afrique de l'Ouest et l'Afrique centrale $(6,16)$. Les analyses recommandées comprennent un examen du frottis sanguin de jour pour les microfilaires de Loa loa.

\section{Délais d'exécution prévus pour les analyses sérologiques}

En l'absence d'immunosuppression, le dépistage de Strongyloides par examen microscopique des selles ou des expectorations pour détecter la présence d'œufs et de parasites ne peut produire un résultat positif que si le patient est atteint d'un fardeau d'infection élevé (e.g. si le patient est symptomatique ou est déjà atteint du syndrome d'hyperinfection). Par conséquent, même si les lignes directrices du CMTMV recommandent le dépistage sérologique et des œufs et des parasites dans les selles, nous ne recommandons pas l'envoi d'œufs et de parasites provenant de selles pour détecter les cas d'asymptomatiques de strongyloïdose-surtout compte tenu de la demande accrue sur les ressources de santé pendant la pandémie de COVID-19. Par conséquent, le test idéal pour dépister les Strongyloides (y compris l'infection asymptomatique) est une analyse sérologique (17). Les analyses sérologiques pour détecter les lgG de $S$. stercoralis sont disponibles dans les laboratoires provinciaux et le délai de traitement des résultats peut être de 10 jours à compter de la réception de l'échantillon de sang par le laboratoire (18). Dans les cas où les résultats sérologiques auront une incidence sur les décisions urgentes en matière de traitement, les laboratoires pourraient être en mesure d'accélérer les résultats des analyses sérologiques. Par exemple, au cours de la dernière troisième vague d'infections en Ontario, le Laboratoire de santé publique de l'Ontario a fourni des résultats accélérés d'analyses sérologiques pour Strongyloides dans les 24 heures suivant la réception par le laboratoire, pour les échantillons marqués comme provenant d'un patient atteint de COVID-19. Une stratégie de laboratoire coordonnée peut aider à préserver des approvisionnements limités en ivermectine tout en veillant à ce que les patients à risque élevé de progression vers l'hyperinfection soient diagnostiqués rapidement.

\section{Conclusion}

COVID-19 légère : Chez les patients atteints de la COVID-19 légère, il n'y a actuellement pas suffisamment de données probantes à l'appui pour l'utilisation de thérapies immunomodulatrices (dexaméthasone et tocilizumab). Si les patients légèrement malades doivent recevoir un traitement immunomodulateur pour la COVID-19 ou pour d'autres indications établies qui ne sont pas liées à la COVID-19, les médecins peuvent utiliser les recommandations du CCMTMV pour orienter les décisions de dépistage et de traitement.

COVID-19 modérée et grave : Les patients qui sont atteints de la COVID-19 modérée ou grave sont susceptibles de recevoir des thérapies immunomodulatrices pour le traitement de la COVID-19 et ils devraient donc être dépistés pour l'exposition ou l'infection à Strongyloides et pourraient nécessiter un traitement avec de l'ivermectine en fonction du risque épidémiologique géographique et du syndrome clinique à Strongyloides soupçonné (I'appendice tableau A1).

\section{Déclaration des auteurs}

$E$. $L$. - A rédigé la première ébauche de l'exposé scientifique E. L., S. R., B. J. L., C. G., N. A., A. M. M. et M. P. - Ont contribué à la conception de l'exposé scientifique

Tous les auteurs ont revu l'exposé de façon critique afin de confirmer son contenu intellectuel important et en ont approuvé la version définitive.

Le contenu de l'article et les points de vue qui y sont exprimés n'engagent que les auteurs et ne correspondent pas nécessairement à ceux du gouvernement du Canada.

\section{Intérêts concurrents}

Aucun.

\section{Remerciements}

La Table de consultation scientifique sur la COVID-19 de l'Ontario est un groupe d'experts scientifiques et de dirigeants du système de santé qui évalue les données probantes émergentes liées à la pandémie de COVID-19 et qui en rend compte afin d'orienter l'intervention de la province de l'Ontario. Le mandat de la Table de consultation scientifique présente des résumés hebdomadaires de données probantes scientifiques pertinentes à la Table de coordination en matière de santé liée à la COVID-19 de la province de l'Ontario, en intégrant les renseignements provenant de tables scientifiques existantes des universités et des organismes de l'Ontario, ainsi que les meilleures données probantes à l'échelle mondiale. La Table scientifique résume ses conclusions à l'intention de la Table de coordination en matière de santé et du public dans des exposés scientifiques. 
Les auteurs tiennent à remercier $\mathrm{P}$ Juni, $\mathrm{M}$ Mclntyre, $\mathrm{S}$ Patel et N Stall pour leur contribution au dossier scientifique de la Table consultative scientifique de l'Ontario, "Traitement à I'ivermectine pour l'infection à Strongyloides chez les patients atteints de COVID-19 ».

Le groupe de travail sur les lignes directrices en matière de pratique clinique pour les médicaments et les produits biologiques est un groupe de médecins et de scientifiques qui possèdent une expertise reconnue en médicaments, en produits biologiques et en soins cliniques. Le groupe de travail évalue les données scientifiques existantes, l'épidémiologie de la maladie, la disponibilité de médicaments et les problèmes de mise en œuvre en vue d'élaborer des lignes directrices en matière de pratique clinique pour le traitement de la COVID-19 au moyen de médicaments et de produits biologiques. Le groupe de travail rend compte de ses conclusions au public et à la Table scientifique. Ses conclusions sont aussi résumées dans des exposés scientifiques.

Les auteurs remercient M. Deilgat et P. Huston pour leurs commentaires utiles et leur correspondance concernant ce manuscrit, étant donné la nature urgente de ce sujet en lien avec le nombre croissant de cas de COVID-19 au Canada.

\section{Financement}

Aucun.

\section{Références}

1. Merck Canada. Monographie de produit. Stromectol comprimés d'ivermectine. 2020. https://www.merck.ca/ static/pdf/STROMECTOL-PM_F.pdf

2. Caly L, Druce JD, Catton MG, Jans DA, Wagstaff KM. The FDA-approved drug ivermectin inhibits the replication of SARS-CoV-2 in vitro. Antiviral Res 2020;178:104787. DOI PubMed

3. Chaccour C, Hammann F, Ramón-García S, Rabinovich NR. Ivermectin and COVID-19: Keeping Rigor in Times of Urgency. Am J Trop Med Hyg 2020;102(6):1156-7. DOI PubMed

4. Clinical Practice Guideline Summary. Recommended Drugs and Biologics in Adult Patients with COVID-19. https:// covid19-sciencetable.ca/sciencebrief/clinical-practiceguideline-summary-recommended-drugs-and-biologics-inadult-patients-with-covid-19-version-2-0/
5. Siemieniuk RA, Bartoszko JJ, Ge L, Zeraatkar D, Izcovich A, Kum E, Pardo-Hernandez $H$, Rochwerg B, Lamontagne $F$, Han MA, Liu Q, Agarwal A, Agoritsas T, Chu DK, Couban R, Darzi A, Devji T, Fang B, Fang C, Flottorp SA, Foroutan F, Ghadimi M, Heels-Ansdell D, Honarmand K, Hou L, Hou X, Ibrahim Q, Khamis A, Lam B, Loeb M, Marcucci M, McLeod SL, Motaghi S, Murthy S, Mustafa RA, Neary JD, Qasim A, Rada G, Riaz IB, Sadeghirad B, Sekercioglu N, Sheng L, Sreekanta A, Switzer C, Tendal B, Thabane L, Tomlinson G, Turner T, Vandvik PO, Vernooij RW, Viteri-García A, Wang Y, Yao L, Ye Z, Guyatt GH, Brignardello-Petersen R, Qasim A, Martinez JP, Cusano E. Drug treatments for covid-19: living systematic review and network meta-analysis. BMJ 2020;370:m2980. DOI PubMed

6. Boggild AK, Libman M, Greenaway C, McCarthy AE; Committee to Advise on Tropical Medicine; au nom du Comité consultatif de la médecine tropicale et de la médecine des voyages (CCMTMV). Déclaration sur la strongyloïdose disséminée : lignes directrices en matière de prévention, d'évaluation et de prise en charge du CCMTMV. Relevé des maladies transmissibles au Canada 2016;42:14-22. DOI

7. Stauffer WM, Alpern JD, Walker PF. COVID-19 and Dexamethasone: A Potential Strategy to Avoid Steroid-Related Strongyloides Hyperinfection. JAMA 2020;324(7):623-4. DOI PubMed

8. Winthrop KL, Mariette X, Silva JT, Benamu E, Calabrese LH, Dumusc A, Smolen JS, Aguado JM, Fernández-Ruiz M. ESCMID Study Group for Infections in Compromised Hosts (ESGICH) Consensus Document on the safety of targeted and biological therapies: an infectious diseases perspective (Soluble immune effector molecules [II]: agents targeting interleukins, immunoglobulins and complement factors). Clin Microbiol Infect 2018;24 Suppl 2:S21-40. DOI PubMed

9. Lier AJ, Tuan JJ, Davis MW, Paulson N, McManus D, Campbell S, Peaper DR, Topal JE. Case report: disseminated strongyloidiasis in a patient with COVID-19. Am J Trop Med Hyg 2020;103(4):1590-2. DOI PubMed

10. Marchese V, Crosato V, Gulletta M, Castelnuovo F, Cristini G, Matteelli A, Castelli F. Strongyloides infection manifested during immunosuppressive therapy for SARS-CoV-2 pneumonia. Infection 2021;49(3):539-42. DOI PubMed

11. Mafort TT, Reis LV, Faria LF, Pinto BM, Silva RV, Miranda CS, Oliveira JG. Alveolar hemorrhage secondary to infection by Strongyloides stercoralis in immunosuppressed patientcase report. Am J Respir Crit Care Med. 2017;195:A5586 (conference abstract). https://www.atsjournals.org/ doi/abs/10.1164/ajrccm-conference.2017.195.1_ MeetingAbstracts.A5586

12. Santé Canada. Rapport de pénurie de médicaments pour STROMECTOL (modifié 2021-04-27; accédé 2021-02-09). https://www.penuriesdemedicamentscanada.ca/ shortage/131914

13. Buonfrate $D$, Bisanzio $D$, Giorli $G$, Odermatt $P$, Fürst $T$, Greenaway C, French M, Reithinger R, Gobbi F, Montresor A, Bisoffi Z. The Global Prevalence of Strongyloides stercoralis Infection. Pathogens 2020;9(6):E468. DOI PubMed 
14. Brown KA, Stall NM, Joh E, Allen U, Bogoch II, Buchan SA, Daneman N, Evans GA, Fisman DN, Gibson JL, Hopkins J, Van Ingen T, Maltsev A, McGeer A, Mishra S, Razak F, Sander B, Schwartz B, Schwartz K, Siddiqi A, Smylie J. Jüni P on behalf of the Ontario COVID-19 Science Advisory Table. A Strategy for the Mass Distribution of COVID-19 Vaccines in Ontario Based on Age and Neighbourhood. Science Table - COVID-19 Advisory for Ontario; (modifié 2020-09-23; accédé 2021-02-26). DOI

15. Buonfrate D, Salas-Coronas J, Muñoz J, Maruri BT, Rodari P, Castelli F, Zammarchi L, Bianchi L, Gobbi F, Cabezas-Fernández T, Requena-Mendez A, Godbole G, Silva R, Romero M, Chiodini PL, Bisoffi Z. Multiple-dose versus single-dose ivermectin for Strongyloides stercoralis infection (Strong Treat 1 to 4): a multicentre, open-label, phase 3, randomised controlled superiority trial. Lancet Infect Dis 2019;19(11):1181-90. DOl PubMed
16. Centers for Disease Control and Prevention. Parasites Loaisis. Atlanta (GA): CDC; (modifié 2015-01-20). https://www.cdc.gov/parasites/loiasis/index.html

17. Dong MD, Karsenti N, Lau R, Ralevski F, Cheema K, Burton L, Klowak M, Boggild AK. Strongyloidiasis in Ontario: performance of diagnostic tests over a 14-month period. Travel Med Infect Dis 2016;14(6):625-9. DOI PubMed

18. Public Health Ontario. Laboratory Services Test Information Index. Strongyloides Serology. PHO; (modifié 2020-07-20). https://www.publichealthontario.ca/en/laboratory-services/ test-information-index/strongyloides-serology

\section{Appendice : Recommandations thérapeutiques}

Pour les recommandations thérapeutiques, nous avons utilisé les définitions suivantes sur la gravité de la COVID-19:

Patients gravement malades : Patients nécessitant un soutien respiratoire et circulatoire, y compris de l'oxygène à haut débit par voie nasale, la ventilation non invasive, la ventilation mécanique invasive ou l'oxygénation de la membrane extracorporelle. Ces patients sont habituellement gérés dans un milieu de soins intensifs.
Patients modérément malades : Patients qui ont récemment besoin d'oxygène supplémentaire à faible débit. Ces patients sont habituellement gérés dans les services d'hospitalisation.

Patients légèrement malades : Patients qui n'ont pas besoin d'oxygène supplémentaire nouveau ou supplémentaire en fonction de leur état de base, de liquides intraveineux ou d'un autre soutien physiologique. Ces patients sont habituellement gérés dans un milieu ambulatoire ou externe. 


\section{Tableau A1 : Recommandations pour le dépistage et le traitement du Strongyloides chez les patients qui sont candidats à recevoir des thérapies immunomodulatrices pour le traitement de la COVID-19 (patients modérément ou gravement malades)}

\section{Catégorie de risque \\ épidémiologique \\ géographique pour \\ l'exposition et l'infection à Strongyloides}

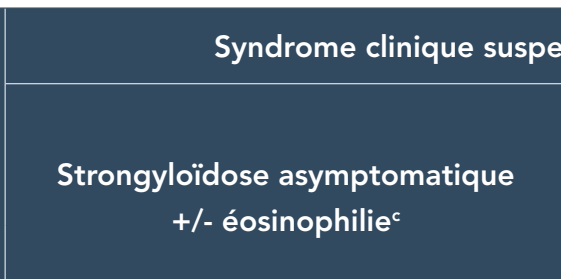

Envoyer la sérologie

Si un résultat sérologique accéléré est attendu :

Aucun traitement empirique à l'ivermectine

n'est requis (ne traiter que si les analyses donnent un résultat positif ou indéterminé) ${ }^{h}$

Si un résultat sérologique accéléré $n$ 'est pas disponible :

Si le patient est cliniquement stable, attendre les résultats de la sérologie (ne traiter que si l'analyse est positive ou indéterminée)

Si le patient est cliniquement instable, envisager un traitement empirique en attendant les résultats de la sérologie (ivermectine $200 \mu \mathrm{g} / \mathrm{kg} \mathrm{PO} \times 1$ )

Si la sérologie est positive ou indéterminée et qu'une $2^{\mathrm{e}}$ dose est administrée, prévoir la dose suivante à un intervalle de 14 jours, à moins qu'il y ait des préoccupations au niveau de l'adhérence

Risque modéré

(naissance ou résidence ou voyage de longue durée $e^{e}$ dans les pays méditerranéens, au Moyen-Orient, en Afrique du Nord, dans le sous-continent indien, en Asie, en Amérique centralef)

\section{Faible risque $^{d}$}

(naissance, résidence ou voyage de longue durée en Australie, en Amérique du Nordg ou en Europe de l'Ouest)

\section{Envoyer la sérologie}

Aucun traitement empirique à l'ivermectine n'est requis (ne traiter que si les analyses donnent un résultat positif ou indéterminé) ${ }^{h}$
Strongyloïdose intestinale simple Consulter un spécialiste des maladies infectieuses et de la médecine tropicale

Envoyer la sérologie et les OP provenant des selles Traitement empirique en attente d'un dépistage (ivermectine $200 \mu \mathrm{g} / \mathrm{kg}$ PO $\times 1$ )

Si la sérologie est positive ou indéterminée et qu'une $2^{\mathrm{e}}$ dose est administrée, prévoir la dose suivante à un intervalle de 14 jours, à moins qu'il y ait des préoccupations au niveau de l'adhérence
Envoyer la sérologie ${ }^{h}$, les OP provenant des selles et les OP provenant des expectorations (+/- autres liquides ou tissus corporels pour examen des larves)

Traitement empirique en attente des résultats (ivermectine $200 \mu \mathrm{g} / \mathrm{kg} \mathrm{PO} \mathrm{q}$ $24 \mathrm{~h}$ jusqu'à l'obtention de résultats d'analyse négatifs)

Envisager l'ajout d'un traitement empirique à l'albendazole

Abréviations : COVID-19, maladie du coronavirus 2019; OP, œufs et parasites; USI, unité de soins intensifs

${ }^{a}$ Consulter un expert en maladies infectieuses ou en médecine tropicale avant d'administrer l'ivermectine si le patient provient d'une région endémique pour le Loa loa

bes doses d'ivermectine en fonction du poids devraient être arrondies à la taille du comprimé entier le plus près (3 mg)

c La plupart des patients atteints de COVID-19 aiguë feront probablement partie de cette catégorie de maladie à Strongyloides soupçonnée. La strongyloïdose chronique est souvent associée à

l'éosinophilie, mais l'éosinophilie peut ne pas être présente lorsque le patient progresse vers une maladie symptomatique ou disséminée

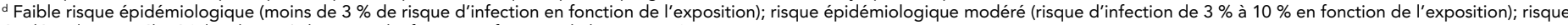
épidémiologique élevé (plus de $10 \%$ de risque d'infection en fonction de l'exposition)

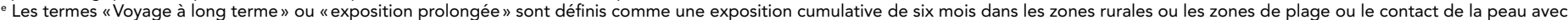

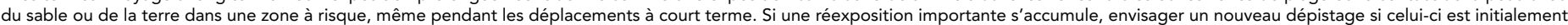
négatif

${ }^{\mathrm{f}}$ Les régions d'Amérique centrale situées à l'extérieur du Mexique peuvent présenter un risque plus élevé que modéré

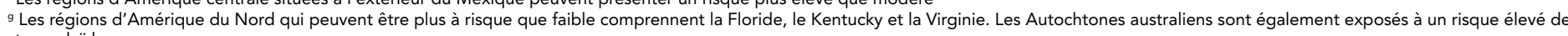
strongylö̈dose

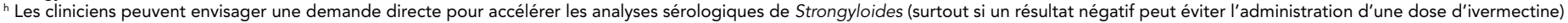

